\title{
Conocimientos y actitudes de la pareja hacia la práctica de la planificación familiar en la Selva del Perú
}

\author{
Doris de la Cruz-Peñarán, $M$ en $C{ }^{(1)} A$ na Langer-G las, $M C^{,}{ }^{(2)}$ Bernardo Hernández-Prado, $\mathrm{Dr}$ en $C,{ }^{(3)}$ \\ Gustavo $\mathrm{F}$ González-Rengijo, D r en C. ${ }^{(4)}$
}

\begin{abstract}
De la Cruz-Peñarán D, Langer-GlasA, Hernández-Prado B, González-Rengijo GF. Conocimientos y actitudes de la pareja hacia la práctica de la planificación familiar en la Selva del Perú. Salud Publica Mex 2003;45:461-471.

El texto completo en inglés de este artículo está disponible en: http://www.insp.mx/salud/index.html
\end{abstract}

\section{Resumen}

Objetivo. Investigar la asociación entre los conocimientos y actitudes, y la práctica de la planificación familiar en parejas de la región de la Selva del Perú. Material y métodos. Estudio transversal llevado a cabo en la región de la Selva del Perú (Pucallpa y Tarapoto). Fueron entrevistadas 600 parejas unidas, varones $\geq 15$ años de edad y mujeres entre 15 y 49 años, ambos con residencia mínima de dos años en el lugar de estudio. Se investigó mediante un cuestionario la práctica de la planificación familiar (definida como el uso de métodos anticonceptivos modernos, con un tiempo de uso $\geq 12$ meses), así como los conocimientos y actitudes sobre la planificación familiar. Se realizaron comparaciones de frecuencias a través de Ji cuadrada. La asociación entre las variables sociodemográficas y el conocimiento de métodos anticonceptivos se analizó a través de modelos de regresión logística, la asociación entre las variables sociodemográficas y las actitudes hacia la planificación familiar a través de modelos de regresión lineal, y, para identificar las variables asociadas al uso de métodos anticonceptivos modernos, se hizo uso de un modelo de regresión logística. Resultados. Los hombres con educación primaria o sin escolaridad fueron más proclives a no conocer el uso de métodos anticonceptivos que aquéllos con educación profesional (RM: 2.18, IC 95\%: 1.05, 4.50). Las mujeres cuya pareja era católica tenían una probabilidad mayor de conocer el uso de métodos anticonceptivos que

\section{De la Cruz-Peñarán D, Langer-Glas A,} Hernández-Prado B, González-Rengijo GF. Sex partners' knowledge and attitudes towards family planning in the Peruvian rainforest. Salud Publica Mex 2003;45:461-471.

The English version of this paper

is available at: http://www.insp.mx/salud/index.html

\begin{abstract}
A bstract
Objective.To assess the association of knowledge and attitudes about family planning (FP) with its practice in couples of the Peruvian jungle region. Material and Methods. A cross-sectional study was carried out in the jungle region in Peru (Pucallpa and Tarapoto), among 600 couples. Males were $\geq 15$ years old and females between 15 and 49 years old, who had resided in the area for at least two years. A questionnaire was used to determine FP practices (defined as the use of modern contraceptive methods (MCM) for $\geq 12$ months), as well as attitudes and knowledge regarding FP. Frequencies were compared using the chi-squared test. Logistic regression was used to assess the association between demographic variables and MCM knowledge as well as MCM utilization. The association between sociodemographic variables and attitudes toward MCM was assessed with linear regression models. Results. Males with primary education or with no formal education were twice as likely to have no knowledge about MCM compared with males with professional education (O R:2.18, 95\% Cl: $1.05,4.50)$. Females with a catholic partner were more likely to know about MCM than those whose partners did not practice any religion ( $\mathrm{R}: 0.60,95 \% \mathrm{Cl}: 0.37,0.97)$. Partner's lower education, older age of women, and rural background of males were all factors associated with a less favorable attitude toward FP practices. Couples in which the man did not know about contraceptive methods were more likely
\end{abstract}

(1) Instituto Nacional de Salud Pública (IN SP) Cuernavaca, Morelos, México. Instituto de Investigaciones de la Altura. Universidad Peruana Cayetano Heredia, Lima, Perú.

(2) The Population Council, Directora Regional para América Latina y El Caribe. México, DF, México.

(3) Salud Reproductiva, IN SP, Cuernavaca, Morelos, México.

(4) Instituto de Investigaciones de la Altura, Lima, Perú.

Fecha de recibido: 29 de abril de 2002 - Fecha de aprobado: 22 de mayo de 2003

Solicitud de sobretiros: D ra. D oris de la Cruz Peñarán. Instituto N acional de Salud Pública. Avenida Universidad 655, colonia Santa María A huacatitlán 62508 Cuernavaca, Morelos, México.

Correo electrónico: dlcruzde75@ hotmail.com, bhernand@ correo.insp.mx 
aquéllas cuya pareja no practicaba religión alguna (RM:0.60, IC 95\%:0.37,0.97). La menor educación de la pareja, la mayor edad de la mujer, y la procedencia rural del hombre se asociaron con una actitud menos favo rable hacia la práctica de la planificación familiar. Las parejas en las cuales el hombre no sabía del uso de métodos anticonceptivos fueron más proclives a no practicar la planificación familiar que las parejas en las que el hombre sí lo conocía (RM: 1.57, IC 95: 1.07, 2.30). Las parejas en las que el varón tuvo un nivel de aceptación medio hacia la planificación familiar tuvieron una probabilidad mayor de no practicarla que aquéllas en las que el hombre tuvo un nivel de aceptación alto (RM: 1.90, IC 95\%: 1.03,3.48). Esta probabilidad fue aún mayor cuando el hombre tuvo un nivel de aceptación bajo hacia la planificación familiar (RM: 2.23, IC 95\%: 1.10, 4.51). Conclusiones El conocimiento de métodos anticonceptivos y las actitudes hacia la planificación familiar entre los hombres se asocian con la práctica de ésta en la pareja. Este estudio indica que la aceptación masculina constituye un factor necesario, importante y de gran influencia en la promoción y práctica de la planificación. El texto completo en inglés de este artículo está disponible en: http://www.insp.mx/salud/ index.html

Palabras clave: anticoncepción; parejas sexuales; conocimientos; actitudes y prácticas; hombres; Perú not to use MCM than couples where the man knew about MCM (O R:1.57,95\% C l:1.07,2.30). C ouples where the man had an intermediate level of acceptance towards FP were more likely not to use MCM than those where the man had a high level of acceptance (0 R:1.90,95\% Cl :1.03, 3.48). The strength of association was higher when the man had a low level of acceptance toward PF (O R:2.23,95\% C : $1.10,4.51)$. Conclusions. Knowledge of contraceptive methods and attitudes toward FP among men are associated with the practice of FP by couples. Study findings show that male acceptance of MCM and FP is a necessary, important, and influential factor in FP promotion and practice.The English version of this paper is available at:http://www.insp.mx/salud/ index.html

Key words: contraception; sexual partners; knowledge, attitudes, practice; men; Peru
L a planificación familiar (PF) es uno de los pilares de la salud reproductiva. Dentro del amplio contexto de salud y derechos reproductivos, la PF debe responder a las necesidades de cada individuo y pareja, promoviendo la participación activa del varón y tomando en consideración las actitudes hacia la anticoncepción, así como de la diversidad cultural de las

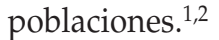

Diversas investigaciones han identificado factores que influyen en la adopción de las prácticas anticonceptivas. Por lo general, los niveles de educación formal, el lugar de residencia y el nivel socioeconómico han sido factores empleados para identificar las poblaciones menos proclives al uso de métodos anticonceptivos (MAC). Hombres y mujeres con mayor escolaridad, mejor nivel ocupacional, mejor nivel socioeconómico y residentes de áreas urbanas ${ }^{*, 3-8}$ muestran una mayor aceptación hacia el uso de MAC. En el Perú, los mayores niveles de uso se concentran en las

\footnotetext{
* De La Cruz D, González GF. Características socioculturales, conocimientos y actitudes hacia la práctica de la planificación familiar en la Costa y Sierra del Perú. XIV Reunión de la Asociación Latinoamericana de Investigadores en Reproducción Humana (ALIRH) Santo Domingo, 7-11 Junio 1995;Documento Santo Domingo, República Dominicana.
}

mujeres con educación secundaria o superior, las que residen en áreas urbanas y las que tienen dos o tres hijos vivos. ${ }^{9,10}$ No obstante, en Pucallpa, en la región de la selva, se ha encontrado que la educación no estuvo asociada al uso de MAC.*

Algunas investigaciones exploran el efecto de otras variables sobre la práctica de la anticoncepción. Por ejemplo, las mujeres y hombres que no participan en programas de PF y tienen conocimientos limitados sobre MAC están menos motivados a usarlos ${ }^{\ddagger, 5,11-18}$ y a preferir familias pequeñas. ${ }^{\ddagger 5,11-15} \mathrm{En}$ un estudio de diversos países dentro de los cuales se incluye a Perú, se mostró que un mayor conocimiento sobre MAC se asocia con un mayor uso de ellos. En este mismo estudio se encontró que la esterilización femenina fue favorecida en el Brasil, mientras que los métodos tradicio-

\footnotetext{
* De La Cruz D, González GF. Variables asociadas al uso de métodos anticonceptivos. XVI Reunión de la Asociación Latinoamericana de Investigadores en Reproducción Humana (ALIRH), Santiago de Chile; Septiembre 24-29 1999;Santiago de Chile, Chile. Documento No publicado.

‡ De La Cruz D, González GF. Características socioculturales, conocimientos y actitudes hacia la práctica de la planificación familiar en la Costa y Sierra del Perú. XIV Reunión de la Asociación Latinoamericana de Investigadores en Reproducción Humana (ALIRH) 1995 junio 7-11; Santo Domingo, República Dominicana. Documento no publicado.
} 
nales, así como la alta fertilidad, fueron preferencias de los hombres en Perú y Haití. ${ }^{16}$

En muchas partes del mundo, los hombres tienen el concepto de que todo lo que tiene que ver con anticoncepción, salud sexual y reproductiva se relaciona exclusivamente con las mujeres. En el Perú, en las ciudades del Cusco y Chimbote, los hombres refirieron que las usuarias principales de los servicios de PF eran las mujeres o, en otras palabras, las personas "débiles." Los hombres se definían a sí mismos como personas fuertes que, por lo tanto, no necesitaban esos servicios. Igualmente, los hombres refirieron que no solicitaban servicios de PF porque el sistema era sólo para las mujeres. ${ }^{19,20}$

Se ha documentado que el hombre continúa ejerciendo influencia, tanto en la decisión de usar MAC, ${ }^{*, 16,21-35}$ como en la decisión sobre el número de hijos que desea tener y en qué momento. ${ }^{22,29,36-40} \mathrm{En}$ diversos contextos, los varones se oponen a que sus esposas practiquen la PF por temor y creencias erróneas sobre los efectos secundarios de los MAC, $, 322-26,31,41,42$ y por temor a la infidelidad de sus parejas. ${ }^{*} \neq, 16,27-29,32-35,41$ Sin embargo, también hay muchos hombres que tienden a apoyar la práctica de la PF al considerarla importante para la salud de la mujer. ${ }^{22,42-45}$ Así, hombres motivados por evitar embarazos fuera del matrimonio apoyan el uso de MAC, como el condón ${ }^{30,42}$ y participan, inclusive, en la elección del método a usar, tomando en cuenta su seguridad y efectos colaterales. ${ }^{30}$ En tanto, en Santa, Perú, se encontró que muchos de los hombres que no usan preservativos en las relaciones extramaritales no lo hacen por considerarlas como "inesperadas". ${ }^{46}$

En el Perú, cuyo contexto sociocultural es muy variado, los hombres se han caracterizado por un patrón cultural "machista", transmitido a través de generaciones, tanto por el padre como por la madre. Esto se ha observado en mayor medida en las regiones de la sierra y selva, ${ }^{\$, 47}$ cuyas características socio-

\footnotetext{
* De La Cruz D, González GF. Características socioculturales, conocimientos y actitudes hacia la práctica de la planificación familiar en la Costa y Sierra del Perú. XIV Reunión de la Asociación Latinoamericana de Investigadores en Reproducción Humana (ALIRH) Santo Domingo, 7-11 Junio 1995;Documento Santo Domingo, República Dominicana.

$\ddagger$ Ali M, Cleland J. Determinants of contraceptive discontinuation in six developing countries: A discrete event history analysis approach. Presented at the Annual Meeting of the Population Association of America, Lousiana, 1996:26-27.

$\S$ Maynard-Tucker G. Men's and women's reproductive and contraceptive decisions: A case study from Highland. Perú, 1994: 3031. Documento no publicado.
}

culturales difieren de las de la región costeña. ${ }^{17,18,35,47,48}$ En la selva, cerca de 70\% de los embarazos en la adolescencia son aceptados, a diferencia de lo que ocurre en las poblaciones de la costa y la sierra donde éste en general no es aceptado. ${ }^{17,49-51}$ Estos factores y sus diferencias seguramente influyen sobre la fecundidad; sin embargo, aún no se les ha dado la debida importancia en la explicación de la práctica anticonceptiva. ${ }^{52}$

Como parte de la actual reforma sectorial, en el Perú se considera al programa de PF ciertamente "prioritario" ${ }^{*}, 53$ La tasa global de fecundidad (TGF) ha disminuido en los últimos 10 años de 4.0 a 2.9 hijos por mujer; sin embargo, esta tendencia no es homogénea en las diferentes regiones del país. En Lima, la capital, la TGF es de 2.0 hijos por mujer; en el resto de la costa es de 2.4; en la región de la sierra es de 3.7 y en la región de la selva es de 3.8 hijos por mujer. ${ }^{9}$ Se atribuye la TGF más alta en la región de la selva a la elevada tasa de embarazos en la adolescencia, y al corto intervalo intergenésico; en esta región, $21.5 \%$ de las adolescentes ya son madres, en comparación a $5.7 \%$ de las adolescentes en Lima. Si bien la prevalencia de uso de anticonceptivos modernos es más baja que en Lima (50\% en la selva y $59 \%$ en la capital), esta prevalencia es aún menor en mujeres adolescentes. ${ }^{9}$

De hecho, en el país, son pocos los estudios que han intentado explicar el efecto que tienen sobre la práctica anticonceptiva los conocimientos y las actitudes, particularmente de los hombres, hacia el uso de MAC modernos en la pareja. Los estudios realizados, por lo general, documentan de manera individual las actitudes hacia la PF, conocimientos y uso de MAC. Estos estudios, sin embargo, no han examinado la asociación entre los conocimientos y actitudes de la pareja hacia la práctica de la $\mathrm{PF}$, particularmente, en la región de la selva del Perú. Además, estos estudios se han llevado a cabo principalmente con datos secundarios.

Los pocos estudios realizados en el país se han concentrado en la ciudad de Lima, la capital. De igual manera, éstos se concentran más en las mujeres, no así en los varones ni en la pareja de manera conjunta, lo que resta importancia al estudio de esta última como una unidad de observación que involucra la comparación de las preferencias de las dos personas que

\footnotetext{
* Chu M, Guerra R. IV Reunión Nacional sobre Población. United States. Agency for International Development (USAID), Asociación Multidisciplinaria de Investigación y Docencia en Población. United Nations Population Fund (UNFPA). Lima, Perú, 1995: 134-136.
} 
participan en el proceso de toma de decisiones reproductivas. Esta comparación es particularmente relevante porque, con frecuencia, el hombre y la mujer no comparten actitudes y metas respecto a la fecundidad y anticoncepción..$^{25,54,55}$

Este artículo tuvo por objetivo principal investigar la asociación entre los conocimientos y actitudes de la pareja en relación con la práctica de la PF en la Selva del Perú. Exploramos estos fenómenos mediante la comparación, tanto de las respuestas de los hombres como de las mujeres, con el fin de identificar las similitudes y diferencias, las cuales pueden ser de importancia con relación a su impacto sobre los resultados. Estos resultados son esenciales y relevantes para explicar la alta TGF de la población de la selva; del mismo modo, este conocimiento es importante para mejorar la calidad de los servicios de PF y reorientar actividades de los programas, para que se adapten mejor a las preferencias y necesidades reales de la población que atienden. Posteriormente, estos datos también pueden ayudar en la evaluación del impacto de los programas de PF.

\section{Material y métodos}

En 1999 se realizó una encuesta en la región de la Selva peruana, en los departamentos de San Martín y Ucayali. Tomando como referencia la TGF promedio de la región de la selva se seleccionó aleatoriamente un departamento con TGF $\geq 4.0$ hijos por mujer (Ucayali), y uno con TGF $<4.0$ hijos por mujer (San Martín). ${ }^{56}$ En cada departamento se seleccionaron aleatoriamente las ciudades de Pucallpa (Ucayali) y Tarapoto (San Martín), y en cada una de ellas dos distritos: Pucallpa y Yarinacocha (Pucallpa), y Tarapoto y Morales (Tarapoto). Todos los sectores que conformaron los distritos fueron considerados en la selección de sujetos.

En cada distrito la selección de viviendas se hizo mediante un muestreo probabilístico sistemático. Es decir, al inicio de cada sector, se tomó al azar una vivienda. A partir de ésta se continuó la selección, dejando una vivienda fuera del estudio e incluyendo la siguiente $(K=1)$ a la derecha del entrevistador. Se entrevistaron 300 parejas unidas y en cada vivienda se entrevistó a todas las parejas que reunieron los criterios de inclusión, y se efectuaron hasta tres visitas cuando era necesario. Así, se incluyeron hombres de edad $\geq 15$ años y mujeres entre 15 y 49 años, ambos con residencia mínima de dos años en el lugar de estudio.

Los datos fueron recolectados por personal (hombres y mujeres) debidamente entrenado. Las entrevistas fueron hechas por separado por una persona del mismo sexo de quien se entrevistaba. Como instrumentos se aplicaron una ficha de selección o tamizaje, para identificar las unidades elegibles (parejas), y un cuestionario estructurado con preguntas abiertas y cerradas, cuyo objetivo era conocer sus características sociodemográficas, su historia reproductiva, el uso y conocimiento de las parejas sobre los MAC y sus actitudes con respecto a la PF. La práctica de la PF fue definida como el uso de MAC modernos por un tiempo mínimo de un año. Usuarios tradicionales de MAC fueron incluidos dentro del grupo de no uso. Respecto al conocimiento, el objetivo principal fue evaluar el conocimiento sobre las características y formas de uso de los métodos, no así si los sujetos conocían de su existencia. Se consideró que el sujeto conocía un MAC si de manera espontánea refería correctamente los aspectos antes mencionados, por ejemplo: describir si se tomaba, o donde se colocaba; lugar donde obtenerlos; ventajas y algunos efectos colaterales. Estas dos variables fueron evaluadas sólo entre aquellos sujetos que refirieron haber escuchado sobre MAC.

La actitud hacia la PF fue medida a través de una escala tipo Likert, ${ }^{57}$ conformada por 20 afirmaciones referidas al número de hijos, uso de la PF y MAC, aborto y contacto con programas de $\mathrm{PF} .{ }^{58}$ En cada afirmación el sujeto indicaba su acuerdo o desacuerdo en una escala del 1 al 5 . El puntaje total de la escala iba de 20 a 100, y puntajes mayores indicaron mayor aceptación hacia la PF. Siguiendo los puntos de corte propuestos por Barrios ${ }^{58}$ esta variable se categorizó en tres niveles de aceptación: alto, medio y bajo. Se consideró que el sujeto alcanzaba un nivel de aceptación bajo cuando obtuvo un puntaje $\leq$ a 51; nivel de aceptación medio, de 52 a 61 puntos, y nivel de aceptación alto, de 62 a 100 puntos. La consistencia interna de esta escala fue aceptable con un coeficiente de alfa de Cronbach de 0.72 .

\section{A nálisis}

Se ejecutaron comparaciones entre las características de los sujetos, según el lugar de estudio (Tarapoto y Pucallpa) y sexo (hombres y mujeres). Considerando que la unidad de análisis fue la pareja, para las variables sobre conocimiento de MAC, actitudes hacia la PF y uso de MAC modernos, se analizó la información proporcionada por cada miembro de ésta.

La asociación entre las variables sociodemográficas y el conocimiento de MAC se analizó por medio de modelos de regresión logística; y la asociación entre las variables sociodemográficas y las actitudes hacia la PF, a través de modelos de regresión lineal. Para identificar las variables asociadas al uso de MAC modernos se empleó la regresión logística. En este último modelo, los sujetos que no hacían uso de MAC, porque deseaban tener hijos, fueron excluidos 
(Pucallpa $n=21$ y Tarapoto $n=36$ ). Como variables independientes se incluyeron las características sociodemográficas, conocimiento de MAC, número de hijos, nivel socioeconómico, actitudes hacia la PF y lugar de estudio.

Las pruebas de máxima verosimilitud y la ji cuadrada de Hosmer y Lemeshow se emplearon para evaluar la bondad de ajuste de los modelos multivariados. Los análisis estadísticos se efectuaron utilizando STATA versión 6.0 .

\section{Resultados}

Se estudió un total de 1200 sujetos, los cuales constituyeron 600 parejas. La tasa de respuesta que se obtuvo fue de $99.84 \%$. En ambas ciudades, la distribución por grupo etáreo fue similar entre las mujeres $(32.17 \pm 8.25$ años) entre los hombres (37.12 \pm 9.20 años). La escolaridad fue mayor entre los hombres que entre las mujeres, aun cuando se encontró diferencia significativa sólo en la ciudad de Tarapoto $(15.33 \%$ hombres vs. $8.67 \%$ mujeres, $p$ : 0008). Más de $60 \%$ de las mujeres de zonas urbanas no trabajaban o se dedicaban a los quehaceres domésticos, en tanto que los varones laboraban sobre todo en trabajos independientes (vendedor) y como empleados. La mayor proporción de sujetos pertenecía a un nivel socioeconómico medio, y en este aspecto no se encontraron diferencias significativas según el sexo y el lugar de estudio. La mayoría de las parejas de Pucallpa (64.33\%) vivían en unión libre (no casados), lo cual contrastaba con las parejas en Tarapoto (38.33\%). En promedio, las parejas tenían $3.11 \pm 1.91$ hijos en Pucallpa y $2.46 \pm 1.52$ hijos en Tarapoto. Tanto hombres como mujeres refirieron practicar más la religión católica en Tarapoto que en Pucallpa, y $15.33 \%$ de las mujeres y $8.33 \%$ de los varones en $\mathrm{Pu}-$ callpa reportaron hablar el dialecto shipibo (cuadro I).

Prácticamente todos los participantes habían oído sobre MAC (97\%). Sin embargo, cuando se les preguntó sobre las características y uso de los métodos que refirieron conocer de manera espontánea, el porcentaje disminuyó hasta la mitad. Entre estos últimos se encontró que las mujeres (particularmente en Pucallpa) tenían un mayor conocimiento de MAC que los hombres (45.92\% mujeres vs. $35.29 \%$ hombres).

Debido a que se encontraron diferencias según el sexo respecto al conocimiento de MAC creamos dos modelos multivariados de regresión logística: uno para los hombres y otro para las mujeres. Los resultados indicaron que, tanto la menor escolaridad del hombre como la de su pareja, y que el hombre hablara el dialecto shipibo, se asociaron con un menor conocimiento de MAC por parte de los hombres. Los mo-

\section{Cuadro I \\ CARActerísticas de hombres y mujeres unidos, en Tarapoto y Pucallpa, Perú, 1999}

\begin{tabular}{|c|c|c|c|}
\hline & Tara & ooto & Pucallpa \\
\hline racterísticas & $\begin{array}{c}\text { \% Hombres } \\
(n=300)\end{array}$ & $\begin{array}{c}\begin{array}{c}\text { \% Mujeres } \\
(n=300)\end{array}\end{array}$ & $\begin{array}{l}\text { \% Hombres \% Mujer } \\
(n=300) \quad(n=300\end{array}$ \\
\hline
\end{tabular}

Promedio de edad $( \pm D E) \quad 36.92 \pm 8.66 \quad 32.43 \pm 7.98 \quad 37.35 \pm 9.73 \quad 31.88 \pm 8.49$

Educación

\begin{tabular}{lrrrr} 
Profesional & 15.33 & 8.67 & 17.67 & 11.33 \\
\hline Técnica & 18.67 & 15.33 & 14.33 & 12.00 \\
\hline Secundaria & 52.33 & 53.00 & 50.00 & 53.33 \\
\hline Primaria/ ninguna & 13.67 & 23.00 & 18.00 & 23.33
\end{tabular}

Ocupación

$\begin{array}{lrrrr}\text { Empleado } & 38.33 & 16.00 & 33.33 & 12.33\end{array}$

\begin{tabular}{lrrrr}
\hline O brero & 11.33 & 2.00 & 9.33 & 1.00 \\
\hline Independiente (ambulante) & 40.67 & 14.33 & 50.00 & 21.67
\end{tabular}

\begin{tabular}{lcccc} 
Campesino & 5.00 & - & 1.67 & - \\
\hline N o trabaja /ama de casa & 4.67 & 67.67 & 5.67 & 65.00
\end{tabular}

Lugar de procedencia

\begin{tabular}{lrrrr} 
Ciudad & 43.67 & 61.67 & 54.00 & 60.00 \\
\hline Pueblo & 36.37 & 31.00 & 31.00 & 27.33 \\
\hline Campo & 17.67 & 7.33 & 15.00 & 12.67
\end{tabular}

Estado civil actual

\begin{tabular}{lllll} 
Casado & 61.67 & 61.67 & 35.67 & 35.67 \\
\hline Conviviente & 38.33 & 38.33 & 64.33 & 64.33
\end{tabular}

Religión

\begin{tabular}{lrrrr} 
N o practica & 6.00 & 6.67 & 28.33 & 14.67 \\
\hline Protestante, otras & 9.33 & 10.00 & 12.00 & 14.33 \\
\hline Católica & 84.67 & 83.33 & 59.67 & 71.00
\end{tabular}

Idioma, dialecto

\begin{tabular}{lrcrr} 
Hablan quechua & 2.33 & 1.00 & 3.00 & 1.33 \\
\hline No hablan quechua & 97.67 & 99.00 & 97.00 & 98.67 \\
\hline H ablan shipibo & 0.33 & - & 8.33 & 15.33 \\
\hline No hablan shipibo & 99.67 & 100.00 & 91.67 & 84.67
\end{tabular}

Nivel socioeconómico

\begin{tabular}{lllll} 
Superior & 21.00 & 22.00 & 21.67 & 20.67 \\
\hline Medio & 51.00 & 53.00 & 48.00 & 42.00 \\
\hline Inferior & 27.67 & 25.00 & 30.33 & 37.33
\end{tabular}

Media de hijos vivos ( \pm DE) $\quad 2.46 \pm 1.52 \quad 3.11 \pm 1.91$

DE: desviación estándar

mios de no conocer sobre MAC entre los varones con educación primaria fueron 2.18 veces mayores que los de aquéllos con educación profesional. Los hombres unidos a mujeres con educación técnica y primaria 
tuvieron mayor oportunidad de conocer MAC modernos hasta en 39 y $42 \%$, respectivamente. Por otro lado, la posibilidad de no conocer sobre MAC entre los varones que hablan shipibo fue 3.52 veces mayor que el de aquellos que no hablan este dialecto. Para el caso de las mujeres, el no tener pareja de religión católica se asoció con un menor conocimiento de MAC (RM: 0.60, IC: 0.37, 0.97). El lugar de estudio no estuvo asociado al conocimiento de MAC por parte de los hombres, ni de las mujeres; no obstante, esta variable se incluyó en los modelos como un potencial confusor (cuadro II).

Las actitudes hacia la PF difirieron también por sexo. Una mayor proporción de las mujeres, en Tarapoto $(23.34 \%)$ y en Pucallpa $(28.92 \%)$ tuvieron un nivel de aceptación más alto que los varones (Tarapoto: $12.98 \%$ y Pucallpa: 9.85\%). Según los resultados del análisis de regresión lineal (cuadro III), los hombres que tuvieron como pareja a una mujer de entre $38.5 \mathrm{y}$ 44.5 años de edad, aquéllos con menor escolaridad, procedentes del campo y residentes de la ciudad de Pucallpa tuvieron una actitud menos favorable hacia la PF. En el grupo de las mujeres, la actitud hacia la PF fue menos favorable entre aquéllas de 44 o más años de edad y con menor nivel de escolaridad. En sentido contrario, la actitud fue más favorable entre aquellas mujeres cuyas parejas no trabajaban o laboraban como campesinos. A diferencia de los hombres, el lugar de estudio no se asoció con las actitudes de las mujeres.

Más de $60 \%$ de los sujetos informaron utlilizar algún MAC moderno, independientemente del tiempo de uso. No se encontraron diferencias en el uso de MAC entre las dos ciudades. Cuando se examinó la prevalencia de uso de MAC modernos en los últimos 12 meses ésta descendía a 40\%, y la distribución continuaba siendo similar entre hombres y mujeres en ambas ciudades. Cabe señalar que 19\% de los varones en Pucallpa manifestaron hacer uso de MAC por indicación médica. El temor a la infidelidad de la pareja (hombres: Pucallpa $43.86 \%$ y Tarapoto $37.50 \%$ ) y la oposición de los maridos (mujeres: Pucallpa $43.10 \%$ ) prevalecieron como razones principales entre las parejas que no usaban MAC modernos.

Puesto que no se encontraron diferencias en el uso de MAC modernos por los dos miembros de la pareja (de acuerdo con el informe de hombres y mujeres), creamos un solo modelo de regresión logística para identificar las variables asociadas a la práctica de la PF. Por otro lado, encontramos que las actitudes de los varones hacia la PF estuvieron significativamente correlacionadas con las actitudes de sus parejas ( $p: 0.000)$; en consecuencia, estas variables no se analizaron de manera simultánea en este modelo.

\section{Cuadro II FACTORES ASOCIADOS CON EL CONOCIMIENTO DE MÉTODOS ANTICONCEPTIVOS MODERNOS EN HOMBRES y mujeres. Tarapoto y Pucallpa, Perú, 1999}

\begin{tabular}{|c|c|c|c|c|}
\hline \multirow[b]{2}{*}{ Variables } & \multicolumn{2}{|c|}{ Hombres } & \multicolumn{2}{|c|}{ Mujeres } \\
\hline & RM* & IC $95 \%$ & RM* & IC $95 \%$ \\
\hline \multicolumn{5}{|l|}{ Edad de la mujer } \\
\hline$<22.0$ & 1 & & 1 & \\
\hline $22.0-25.5$ & 0.94 & 0.471 .90 & 0.51 & 0.251 .02 \\
\hline $25.5-31.0$ & 1.27 & 0.652 .51 & 0.81 & 0.411 .59 \\
\hline $31.0-38.5$ & 1.50 & 0.782 .90 & 0.77 & 0.401 .47 \\
\hline $38.5-44.5$ & 1.60 & 0.773 .35 & 0.70 & 0.341 .43 \\
\hline$\geq-44.5$ & 1.28 & 0.572 .88 & 0.68 & 0.311 .50 \\
\hline \multicolumn{5}{|l|}{ Educación de la mujer } \\
\hline Profesional & 1 & & 1 & \\
\hline Técnica & 0.39 & $0.180 .84^{\ddagger}$ & 0.87 & 0.431 .74 \\
\hline Secundaria & 0.59 & 0.301 .16 & 0.65 & 0.361 .16 \\
\hline Primaria/ ninguna & 0.42 & $0.190 .92^{\ddagger}$ & 0.57 & 0.301 .07 \\
\hline
\end{tabular}

Educación del hombre

\begin{tabular}{llllll} 
Profesional & 1 & & & \\
\hline Técnica & 1.84 & 0.983 .45 & & \\
\hline Secundaria & 1.48 & 0.872 .49 & & \\
\hline Primaria/ ninguna & 2.18 & 1.05 & $4.50^{\ddagger}$ & & \\
& & & & \\
Religión del hombre & & & & \\
No practica & 1 & & 1 & \\
\hline Protestante, otras & 1.77 & 0.873 .59 & 0.62 & 0.321 .21 \\
\hline Católica & 1.40 & 0.862 .29 & $0.60^{\ddagger}$ & 0.37 & 0.97
\end{tabular}

Dialecto shipibo del hombre Hombre que no habla shipibo 1 Hombre que habla shipibo $\quad 3.08 \quad 1.019 .43^{\ddagger}$

Lugar de estudio

\begin{tabular}{|c|c|}
\hline Tarapoto & 1 \\
\hline Pucallpa & 1.23 \\
\hline
\end{tabular}

* RM Razón de momios ajustada por características sociodemográficas de hombres y mujeres, tanto como de su pareja y lugar de estudio $\neq p:<0.05$

Los resultados mostraron que las parejas en las cuales las mujeres tenían entre 25.5 y 31 años, entre 31 y 38.5 años y aquéllas entre 38.5 y 44.5 años de edad fueron más proclives a usar MAC modernos que las parejas en las cuales las mujeres eran menores de 22 años de edad (RM: 0.40, IC 95\%: 0.17, 0.93; RM: 0.27, IC $95 \%: 0.11,0.62$ y RM: 0.34, IC 95\%: 0.13, 0.85, respectivamente). El conocimiento de MAC y las actitudes de los hombres hacia la PF tuvieron un efecto significativo en las probabilidades de no uso de MAC modernos en la pareja (cuadro IV). Las parejas en las cuales 


\section{Cuadro III \\ Factores asociados con UnA actitud favorable O DESFAVORABLE HACIA LA PRÁCTICA DE LA PLANIFICACIÓN FAMILIAR EN HOMBRES Y MUJERES. Tarapoto y Pucallpa, Perú, 1999}

Variable \begin{tabular}{cccc}
\multicolumn{2}{c}{ Hombres } & & \multicolumn{2}{c}{ Mujeres } \\
\cline { 1 - 2 }$\beta^{*}$ & IC $95 \%$ & & IC $95 \%$
\end{tabular}

Edad de la mujer

\begin{tabular}{lllllll}
$<22.0$ & 0 & & 0 & & & \\
\hline $22.0-25.5$ & 0.16 & -3.32 & 3.65 & 1.78 & -1.41 & 4.99 \\
\hline $25.5-31.0$ & -1.88 & -5.21 & 1.45 & -0.93 & -4.01 & 2.14 \\
\hline $31.0-38.5$ & -0.96 & -4.19 & 2.26 & -0.20 & -3.29 & 2.88 \\
\hline $38.5-44.5$ & -3.53 & -7.11 & $0.03^{\ddagger}$ & -2.54 & -5.93 & $0.85^{\ddagger}$ \\
\hline$\geq-44.5$ & -2.35 & -6.26 & $1.56^{\ddagger}$ & -4.41 & -8.18 & $-0.65^{\ddagger}$
\end{tabular}

Educación de la mujer

\begin{tabular}{lcccccc} 
Profesional & 0 & 0 & & & & \\
\hline Técnica & -1.84 & -5.49 & 1.80 & -0.93 & -4.28 & 2.42 \\
\hline Secundaria & -1.87 & -5.00 & 1.24 & -3.54 & -6.41 & -0.67 \\
\hline Primaria/ ninguna & -5.87 & -9.52 & $-2.21^{\ddagger}$ & -6.79 & -10.17 & $-3.41^{\ddagger}$
\end{tabular}

Educación del hombre

Profesional $\quad 0 \quad 0$

\begin{tabular}{lrrrrrr}
\hline Técnica & -1.70 & -4.78 & 1.36 & 0.90 & -1.89 & 3.70 \\
\hline Secundaria & -5.63 & -8.26 & -3.01 & -2.17 & -4.66 & 3.30 \\
\hline Primaria/ ninguna & -12.20 & -15.74 & $-8.66^{\ddagger}$ & -6.93 & -10.25 & -3.60
\end{tabular}

Procedencia del hombre

\begin{tabular}{llll} 
Ciudad & 0 & & \\
\hline Pueblo & -1.12 & -3.05 & 0.79 \\
\hline Campo & -3.23 & -5.74 & $-0.71^{\ddagger}$
\end{tabular}

0 cupación del hombre

\begin{tabular}{llll} 
Empleado & 0 & & \\
\hline O brero & 2.65 & -0.26 & 5.57 \\
\hline Independiente (ambulante) & 1.52 & -0.36 & 3.41 \\
\hline C ampesino & 4.58 & -0.03 & $9.20^{\ddagger}$ \\
\hline No trabaja & 4.26 & 0.52 & $8.00^{\ddagger}$
\end{tabular}

Religión del hombre

\begin{tabular}{llll} 
No practica & 0 & & \\
\hline Protestante, otras & -2.36 & -5.38 & 0.65 \\
\hline Católica & -1.70 & -3.85 & 0.45
\end{tabular}

Estado civil conviviente

$-2.22 \quad-4.06-0.39$

Lugar de estudio:

Tarapoto Pucallpa

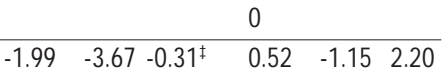

* $\beta$ Coeficientes de regresión lineal ajustados por características sociodemográficas tanto de hombres y mujeres, como de su pareja y lugar de estudio

$\ddagger \mathrm{p}:<0.05$
Cuadro IV

FACTORES ASOCIADOS CON EL NO USO DE ANTICONCEPTIVOS MODERNOS EN PAREJAS UNIDAS. Tarapoto y Pucalla, Perú, 1999

$\begin{array}{lll}\text { RM } & \text { RM* } \\ \text { Crudo } & \text { IC 95\% } & \text { ajustado IC } 95 \%\end{array}$

\begin{tabular}{lllll}
$\begin{array}{l}\text { Edad de la mujer } \\
<22.0\end{array}$ & 1 & & 1 & \\
\hline $22.0-25.5$ & 0.60 & $0.28-1.30$ & 0.77 & $0.32-1.83$ \\
\hline $25.5-31.0$ & 0.44 & $0.21-0.92$ & $0.40^{\ddagger}$ & $0.17-0.93$ \\
\hline $31.0-38.5$ & 0.25 & $0.12-0.52$ & $0.27^{\ddagger}$ & $0.11-0.62$ \\
\hline $38.5-44.5$ & 0.25 & $0.12-0.55$ & $0.34^{\ddagger}$ & $0.13-0.85$ \\
\hline$\geq-44.5$ & 0.44 & $0.19-1.01$ & 0.55 & $0.20-1.48$
\end{tabular}

Edad del hombre

\begin{tabular}{lll}
$<30$ & 1 & \\
\hline $30-44$ & 0.61 & $0.40-094$ \\
\hline$\geq-44$ & 0.65 & $0.39-1.05$
\end{tabular}

Educación de la mujer (años)

$\begin{array}{llll}1.01 & 0.96-1.06 & 1.04 & 0.97-1.11\end{array}$

Educación del hombre (años)

$0.98 \quad 0.94-1.03$

Procedencia del hombre

\begin{tabular}{lll} 
Ciudad & 1 & \\
\hline Pueblo & 1.31 & $0.90-1.92$ \\
\hline Campo & 1.06 & $0.66-1.69$
\end{tabular}

Religión de la mujer

\begin{tabular}{lll} 
No practica & 1 & \\
\hline Protestante, otras & 0.54 & $0.31-0.96$ \\
\hline Católica & 0.73 & $0.35-1.52$
\end{tabular}

Religión del hombre

\begin{tabular}{lll} 
No practica & 1 & \\
\hline Protestante, otras & 0.62 & $0.39-0.99$ \\
\hline Católica & 0.51 & $0.25-1.01$
\end{tabular}

$0.51 \quad 0.25-1.01$

Estado civil conviviente $\quad 1.36 \quad 0.97-1.89$

Dialecto quechua de la mujer $\quad 0.34 \quad 0.06-1.78$

\begin{tabular}{lll}
\hline Número de hijos & 0.89 & $0.80-0.98$
\end{tabular}

No conocimiento de MAC de la mujer $1.14 \quad 0.82-1.59$

\begin{tabular}{lllll}
\hline No conocimiento de MAC del hombre & 1.46 & $1.03-2.06$ & $1.57^{\ddagger}$ & $1.07-2.30$
\end{tabular}

N ivel de aceptación de PF de la mujer

\begin{tabular}{lll} 
Alto & 1 & \\
\hline Medio & 1.15 & $0.77-1.69$ \\
\hline Bajo & 1.28 & $0.69-2.37$
\end{tabular}

Nivel de aceptación de PF del hombre

\begin{tabular}{lllll} 
Alto & 1 & \multicolumn{3}{l}{1} \\
\hline Medio & 1.39 & $0.77-2.52$ & $1.90^{\ddagger}$ & $1.03-3.48$ \\
\hline Bajo & 1.54 & $0.87-2.60$ & $2.23^{\ddagger}$ & $1.10-4.51$
\end{tabular}

Lugar de estudio

\begin{tabular}{lll} 
Tarapoto & 1 & \\
\hline Pucallpa & 1.30 & $0.93-1.82$
\end{tabular}

$R^{2}: 36.19, p>0.0006, P$. Hosmer-Lemshow: 0.78 (modelo ajustado)

MAC :métodos anticonceptivos

PF:planificación familiar

* RM: Razón de momios ajustados por edad y educación de la mujer número de hijos, no conocimiento de MAC por el hombre y nivel de aceptación de la PF del hombre. No se incluyen en el modelo los sujetos que quieren tener hijos

$\neq p:<0.05$ 
los hombres no conocían sobre MAC tuvieron una posibilidad 1.57 veces mayor (RM: 1.57, IC 95\%: 1.07, 2.30) de no usar anticonceptivos modernos que aquéllas en las que el hombre sí los conocía. De manera más acentuada, las parejas cuyos integrantes masculinos tenían un nivel de aceptación medio hacia la PF tuvieron una posibilidad 1.90 veces mayor (RM: 1.90 , IC $95 \%: 1.03,3.48$ ) de no usar MAC modernos que aquéllas en las que el hombre tenía un nivel de aceptación alto; y tuvieron una posibilidad 2.23 veces mayor de no usar MAC modernos (RM: 2.23, IC 95\%: 1.10, 4.51) cuando los hombres tenían un nivel de aceptación bajo hacia la PF. La variable lugar de estudio fue controlada como potencial confusor; en tanto, fue excluida de este modelo debido a que no mostró efecto alguno.

\section{Discusión}

Estos resultados indican que en la Selva del Perú la oposición de los hombres a los MAC tiene un efecto importante en su uso en la pareja; consecuentemente, estas parejas están expuestas a tener embarazos no planeados y posiblemente no deseados. Estos grupos, sin embargo, podrían beneficiarse de servicios de PF diseñados acorde con sus necesidades. Por ejemplo, la asociación del uso de MAC modernos por la pareja con el conocimiento de métodos por parte del hombre sugiere que es necesario reforzar el componente de información dirigida a los hombres, y garantizar que se dé el conocimiento necesario. En este sentido, otros estudios son congruentes con nuestros resultados al señalar que, cuanto mayor es el conocimiento de MAC, mayor es su uso, particularmente entre los hombres. ${ }^{13,15,25}$

Es evidente que tanto hombres como mujeres necesitan de mayor información sobre MAC, particularmente modernos, pues como mostró este estudio, si bien más de $90 \%$ de hombres y mujeres refirieron conocer sobre $\mathrm{MAC}$, al indagar sobre sus características y uso, este porcentaje descendió $50 \%$. Por otro lado, en las parejas que refirieron no usar MAC modernos, los hombres en su mayoría lo hacían por temor a la infidelidad de su pareja. Por su parte, las mujeres en $\mathrm{Pu}$ callpa no usaban MAC por oposición de sus maridos quienes, a su vez, asociaban el uso de anticonceptivos con la infidelidad, mientras las mujeres en Tarapoto no los usaban por temor a los efectos colaterales. En este contexto, los programas de PF podrían dirigir sus estrategias a mejorar el conocimiento de MAC entre los hombres y mujeres, pero estas intervenciones también deberían tener por objetivo corregir la información errónea acerca de los efectos secundarios de estos métodos, así como trabajar sobre los aspectos culturales que subyacen a la asociación entre anticoncepción e infidelidad.

Llama la atención que los hombres continúen ejerciendo mayor influencia que las mujeres en cuanto a la anticoncepción de la pareja, según lo demuestran diversos estudios; $;, \neq, 3,14,23-32,36,40,41,59,60$ mientras que las actitudes del marido no se ven influidas en forma similar por las de su mujer. ${ }^{3}$ Estos hallazgos son congruentes con los de nuestro análisis. Se ha visto que aún cuando las actitudes de la pareja hacia la PF se encontraron correlacionadas, las de las mujeres no se asociaban al uso de MAC por la pareja del mismo modo que las actitudes de los varones.

Si se reconoce esta influencia, podríamos suponer que el incremento en el uso de anticonceptivos modernos en la pareja, que se ha observado en los últimos años, podría atribuirse a los cambios en las actitudes o percepciones principalmente de los hombres hacia la PF. En este contexto, se podría decir que el éxito de lograr una familia más pequeña dependerá de la influencia que el hombre ejerza sobre la conducta reproductiva de la pareja.

Diversos estudios han mostrado una fuerte asociación entre la educación de la pareja (especialmente de los varones) y el uso de MAC, al encontrar que los hombres con mayor escolaridad consideran que son tan responsables como las mujeres de prevenir los embarazos. ${ }^{5-8,61}$ En contraste, este estudio indicó que la educación no afecta directamente y de manera significativa el uso de MAC modernos por la pareja. Sin embargo, es posible que la educación esté afectando indirectamente la práctica de la $\mathrm{PF}$, ya que la menor escolaridad de los sujetos se asoció con una actitud menos favorable hacia ella.

Igualmente, se ha descrito que el nivel socioeconómico influye sobre las prácticas anticonceptivas,; ${ }^{3}$ no obstante, en este estudio no se encontró asociación entre el nivel socioeconómico y el uso de MAC modernos por hombres y mujeres. Probablemente, la falta de esta relación se debió a que el nivel socioeconómico fue relativamente homogéneo en las comunidades estudiadas.

* De La Cruz D, González GF. Características socioculturales, conocimientos y actitudes hacia la práctica de la planificación familiar en la Costa y Sierra del Perú. XIV Reunión de la Asociación Latinoamericana de Investigadores en Reproducción Humana (ALIRH) Santo Domingo, 7-11 Junio 1995;Documento Santo Domingo, República Dominicana.

‡ Ali M, Cleland J. Determinants of contraceptive discontinuation in six developing countries: A discrete event history analysis approach. Presented at the Annual Meeting of the Population Association of America, Lousiana, 1996:26-27. 
Este artículo contribuye al estudio de la planificación familiar desde una perspectiva de género, ya que los datos empíricos que presentamos permiten reflexionar sobre el significado de las diferencias en las actitudes de los hombres y mujeres. Estudiar a la pareja nos permite plantear que las diferencias encontradas no sólo se asocian con cuestiones biológicas (ser hombre o ser mujer), sino también que se derivan de las desigualdades social y culturalmente determinadas en los comportamientos y actitudes de hombres y mujeres.

Por otro lado, no podemos olvidar que, objetivamente, existen más MAC para la mujer que para el hombre y que esta realidad contribuye a la percepción que tiene la población masculina de que a la mujer "es a quien le corresponde la responsabilidad de cuidarse". La complejidad e interacción de estos factores nos permite afirmar que las diferencias encontradas son culturales y se derivan de construcciones sociales sobre el significado de ser hombre o ser mujer en el ámbito de la reproducción.

Con base en nuestros resultados es posible recomendar otras investigaciones: a) intervenciones para involucrar a los hombres en los programas de PF y lograr una participación más activa de los mismos, pues una consecuencia de un enfoque dirigido únicamente a la mujer ha sido la de que muchos de los hombres vean con desconfianza las actividades de PF, y consideren que éstas están dirigidas a socavar su autoridad en la familia y, consecuentemente, se opongan a la práctica de la PF al asociarla con la infidelidad; b) el papel de los factores socioculturales en la determinación de las actitudes de las parejas, especialmente de los hombres, hacia la práctica de la PF; c) actitudes de las parejas adolescentes frente a la PF, ya que son un grupo prioritario, según lo demuestran nuestros resultados al señalar particularmente que las mujeres menores de 22 años de edad tienen una menor probabilidad de usar MAC modernos. Finalmente, sería esencial que se ampliaran investigaciones sobre la calidad de los servicios de planificación familiar.

También, es necesario recurrir a otras formas de abordajes como los métodos cualitativos, que permitan comprender de manera más específica el fenómeno en estudio, e indagar los significados e interpretaciones propias de los sujetos. Los métodos cualitativos podrían atenuar limitaciones propias de los estudios cuantitativos, como sesgos de memoria o de selección. En el caso de este estudio consideramos haber contrarrestado estos efectos con preguntas de reforzamiento, entrevistas a los sujetos por personas de su mismo sexo y el haber obtenido una tasa de respuesta de $99.84 \%$.

En general, pese a las limitaciones de este diseño, los resultados ofrecen importantes aportes. En el Perú, donde la práctica de la PF continúa siendo baja y la TGF alta, en especial en la región de la Selva, la aceptación masculina constituye un factor necesario, importante y de gran influencia para la promoción y práctica de la anticoncepción. Este estudio sugiere que la calidad de los servicios de PF debe ser mejorada principalmente en aquellos componentes que podrían lograr mayor conocimiento y mejor actitud entre los hombres con relación a los MAC modernos.

\section{Agradecimientos}

Agradecemos el apoyo de The Population Council de Perú, y de la Organización Mundial de la Salud, Programa de Reproducción Humana. Agradecemos la participación de la licenciada Ana Florián, en la conducción del trabajo de campo.

\section{Referencias}

1. Pérez G, Gotilla A, Gil F, Macchetto A, N úñez A, C ravioto $M$ et al. La planificación familiar en el contexto de la Salud Reproductiva. México, DF: Espiral Editora, 1998:15-20.

2. Fathalla M. Reproductive health in the world:Two decades of progress and the challenge ahead. En:W orld Health 0 rganization/ HRP. Reproductive Health:A key to a brighter future. Biennial Report, 1990-1991. G inebra:W HO, 1992.

3. D hamalingam A.The social context of family planning in a South Indian village. Int Fam Plann Perspect 1995;21:98-103.

4. Pick W M, 0 bermeyer CM. U rbanization, household composition and the reproductive health of women in a South African city. Soc Sci Med 1996;43(10):1431-1441.

5. Mahmood N, Ringheim K. Knowledge, approval and communication about family planning as correlates of desired fertility among spouses in Pakistan. Int Fam Plann Perspect 1997;23:122-129.

6. Castro T, Juárez F.The impact of women's education on fertility in Latin A merica: Searching for explanations. Int Fam Plann Perspect 1995;21:52-57,80.

7. D ang A. D ifferentials in contraceptive use and method choice in Vietnam. Int Fam Plann Perspect 1995;21:2-5.

8. Petro-N ustas W. Men's knowledge of and attitudes toward birthspacing and contraceptive use in Jordan. Int Fam Plann Perspect 1999;25(4):181-185.

9. Instituto $\mathrm{N}$ acional de Estadística e Informática. Encuesta $\mathrm{N}$ acional de Demografía y Salud. Programa de Encuestas de Demografía y de Salud Familiar. Lima, Perú: Ministerio de Salud, 2000:47-57,62-67.

10. Instituto $\mathrm{N}$ acional de Estadística e Informática. Salud reproductiva, pobreza y condiciones de vida en el Perú. Lima, Perú: Ministerio de Salud, 1999:338-340.

11. Bounlay M,Valente T.The relationship of social affiliation and interpersonal discussion to family planning knowledge, attitudes and practice. Int Fam Plann Perspect 1999;25(3):112-118,138.

12.0 bby $\mathrm{C}$. Family planning attitudes and use in $\mathrm{N}$ igeria. $\mathrm{A}$ factor analysis. Int Fam Plann Perspect 1999;25(2):86-91.

13. Mi Kim Y, Kols A, Mucheke S. Informed choice and decision-making in family planning counseling in Kenya. Int Fam Plann Perspect 1998;24(1):4-11,42. 
14.Amin R, LiY,A hmed A. W omen's credit programs and family planning in rural Bangladesh. Int Fam Plann Perspect 1996;22:158-162. 15. Rutenberg N, Ayad M, O choa LH, W ilkinson M. Knowledge and use of contraception. Columbia (MD): Institute for Resource Development, 1991; D HS Comparative Studies, 6:67.

16. Loayza E. Male fertility, contraceptive use, and reproductive preferences in Latin América. En: Panthalides E, Ed. Men family formation and reproduction. Buenos Aires, Argentina: Centro de Estudios de Población, 1998:13-15.

17. Magnani R, Seiber E, Gutiérrez E,Vereau D. Correlates of sexual activity and condom use among secondary-school students in urban Peru. Stud Fam Plann 2001;32(1):53-66.

18. Leon F, Monge R, Zumarán A, G arcía I, Ríos A. Length of counselling sessions and the amount of relevant information exchanged: Study in Peruvian clinics. Int Fam Plann Perspect 2001;27(1):28-33, 46. 19.Valente T, Paredes P, Poppe P. Matching the message to the process: The relative ordering of knowledge, attitudes, and practices in behavior change research. Human Comm Res 1998;24(3):366-385.

20. Cobián E, Reyes S. Masculine perceptions of family planning and reproductive health needs and services:A qualitative study in Chimbote. En: Investigación de operaciones en planificación familiar y salud reproductiva: conceptos y casos. Lima, Perú: Universidad Peruana Cayetano Heredia, Instituto de Estudios de Población, 1998:123-125. 21. Schuler SR, Hashemi SM, Jenkins AH. Bangladesh's family plannings success story: A gender perspective. Int Fam Plann Perspect 1995;21(4):132-137,166.

22. D onovan P. In N igeria, traditions of male dominance favor large families, but some men report having fewer children. Int Fam Plann Perspect 1995;21(1):39-40.

23. Barnett B. El uso de la planificación familiar es a menudo una decisión familiar. N etwork en Español 1998;22(1):11-15.

24. Robey B, Ross J, Brushan I. Cómo atender la necesidad insatisfecha: nuevas estrategias. Pop Rep Septiembre 1996;2:8-10.

25. Bankole A, Singh S. Couples' fertility and contraceptive decision-making in developing countries: Hearing the man's voice. Int Fam Plann Perspect 1998;24(1):15-24.

26. Guzmán S, Snow R,Aitken I. Preferences for contraceptive attributes:Voices of women in Ciudad Juárez, México. Int Fam Plann Perspect 1997;23:52-58.

27. Bankole A.The impact of mass media family planning promotion on contraceptive behavior in N igeria. Calverton (MD): Macro International, 1994; D HSW orking Papers N 0.11.

28. Fort A. Investigando el contexto social de la fecundidad y la planificación familiar: estudio cualitativo en el Perú. Perspect Int Plan Fam 1990:1-8.

29. Figueroa J, Liendro E. La presencia del varón en la salud

reproductiva. En: Centro de Investigación Materno Infantil de Campinas. Ciencias sociales y medicina. Actualidades y perspectivas latinoamericanas. Campinas, Brasil: U niversidad Estadual de C ampinas, 1995:193-226

30. Ezeh AC. The influence of spouses over each other's contraceptive attitudes in G hana. Stud Fam Plann 1996;22(4):571-593.

31. Casterline J, Pérez E, Biddlecom A. Factors underlying unmet need for family planning in the Philipines. Stud Fam Plann 1997:28(3):173-191. 32. Ravindran T. User 's perspectives on fertility regulation methods. Econ Poli W kly 1993;21(4):45-46.

33. McC auley A, Robey B, Blanc A, G eller J. O pportunities for women through reproductive choice. Baltimore (MD): Johns Hopkins School of Public Health, Population Information Program. Pop Rep 1994;Series M, N umber 12: 40.

34. Saravia C, A polinario H, Morales R, Reynoso B, Salinas V.The condom access route for adolescents of Lima, Cusco and Iquitos. En: $N$ uevos retos: investigaciones recientes sobre salud sexual y reproductiva de los jóvenes en el Peru: Lima, Perú: RED ESS Jóvenes, 1999:95-116.
35. Schuler S. Gender and community participation in reproductive health projects: Contrasting models from Perú and G hana. Reprod Health Matters 1999:7(14):144-157.

36. Dirección General de Salud Reproductiva. La perspectiva de género en la salud reproductiva. México, DF:SSA, 1995:10-11.

37. McC auley A, Robey B, Blanc A, G eller Y. O portunidades para la mujer mediante la libre decisión reproductiva. Pop Rep Julio 1994;Serie M N umber 12:17-19.

38. Lynn C. Participación del hombre después del embarazo. N etwork en Español 1997;18(4):22-25.

39. G reen $C P, C$ ohen SL, Belhadj EL, D anforth $N$, Jezowski T, Beyond C. Los hombres y la salud de la reproducción. N etwork, en español. 1998;18(3):4-6, 12-14.

40. Schuller S, Choque M. Rance S. information, mistrust and mistreatment: Family planning among Bolivian market women. Stud Fam Plann 1994:25(4):211-221.

41. Rutenberg $\mathrm{N}, \mathrm{W}$ atkins $\mathrm{S}$. Conversation and contraception in $\mathrm{N}$ yanza Province, Kenya. N ueva 0 rleans (LA): 1996:30-31.

42. Arias R.A puro valor mexicano. Connotaciones del uso del condón en hombres de la clase media de la ciudad de México. Coloquio Latinoamericano sobre Varones, Sexualidad y Reproducción. México, DF: 1995.

43. Biddlecom A, C asterline J, Pérez A. Spouses' views of contraception in the Philippines. Int Fam Plann Perspect 1997;23:108-115.

44. Paulson S. Los hombres en Bangladesh desempeñan un papel en la planificación familiar. N etwork en Español 1992;24(18):20-21.

45. Robey B, Rutstein SO, Morris L, Blackburn R. La Revolución reproductiva: nuevos resultados de las encuestas. Pop Rep 1992; 20(4):1-43.

46. León F, C hu M. Investigación de operaciones en planificación familiar y salud reproductiva: conceptos y casos. Lima, Perú:The Population Council del Perú, 1998:133-145.

47. Sobrevilla L,Alcántara E, Garther E. N acer y morir en la pobreza. Lima: Instituto de Estudios de Población, 1987.

48. Foreit J, $G$ arate $M$, Brazzoduro $A$, G uillén $F$, Herrera $M, C$ ardoso $C$ et al.A comparision of the performance of male and female $C B D$ distributors in Perú. Stud Fam Plann 1992;23(1):58-62. 49. González GF. La adolescencia en el Perú. Lima, Perú: Instituto de Investigaciones de la Altura, Universidad Peruana Cayetano Heredia, 1994.

50. Hammer P, Escobar M. Evaluation of sociocultural factors affecting reproductive and sexual health of rural adolescents. Lima, Perú: University of N orth Carolina at Chapel Hill, School of Medicine, Program for International Training in health (IN TRAH), 1999:33-63. 51. Sebastiani A, Segil E.W hat the male and female adolescents of Lima do, think, and feel concerning sexual and reproductive health. En: $N$ uevos retos: investigaciones recientes sobre salud sexual y reproductiva de los jóvenes en el Perú. Lima, Perú: RED ESS Jóvenes, 1999:43-60

52. Paolisso M, Leslie J. Meeting the changing health needs of women in developing countries. Soc Sci Med 1995;40(1):55-65.

53. Ministerio de Salud. Seminario Internacional Reforma del Sector Salud. Programa de fortalecimientos de Servicios de Salud y el Proyecto Generación de Capacidades en el Ministerio de Salud. Lima, Perú: Ministerio de Salud, 1997:3-7, 9-29.

54. Bankole A. Desired fertility and fertility behavior among the Yoruba of $N$ igeria: A study of couple preferences and subsequent fertility. Pop Stud 1995;49(2):317-328.

55. Figueroa J. Algunas reflexiones sobre la representación social de la sexualidad femenina. Rev N ueva Antropol Mex 1994;(43):141-166. 56. Instituto $\mathrm{N}$ acional de Estadística e Informática. Encuesta $\mathrm{N}$ acional de Demografía y Salud (EN DES). Programa de Encuestas de Demografía y de Salud Familiar. Lima, Perú: Ministerio de Salud, 1996:12-14, 43-46. 
57. Silva A. Métodos cuantitativos en psicología. Un enfoque metodológico. Las escalas de actitudes y la sociometría en la práctica psicológica México, D F:Trillas, 1992:777-787.

58. Barrios C, G onzález G. Conocimientos y actitudes de los varones hacia la planificación familiar en la ciudad de Lima. (Tesis de Bachiller). Lima, Perú: Universidad Peruana Cayetano Heredia, 1990.

59. Palma Y, Figueroa J, Cervantes A. D inámica del uso de métodos anticonceptivos en México. Rev Mex Sociol 1990;(1):51-81.
60. Lloyd C. Family and gender issues for population policy. N ueva York (N Y): Population Council, 1993; Research Division W orking Paper:

41-42.

61. Mensch B,A rends-Kuenning M, Jain A, G árate R.Avoiding unintended pregnancy in Perú: D oes the quality of family planning services matter? Int Fam Plann Perspect 1997;23:21-27. 